\section{Trapping influenza neuraminidase}

\author{
By Lev Osherovich, Senior Writer
}

Researchers at The University of British Columbia have used a mechanism-based approach to design irreversible inhibitors of influenza virus neuraminidase that could have activity against flu strains resistant to marketed inhibitors of the target. ${ }^{1}$ The new molecules are being developed by CDRD Ventures Inc., the venture arm of The Centre for Drug Research and Development, a public-private partnership that commercializes discoveries from Canadian researchers.

Influenza virus uses neuraminidase to cleave sugars from host glycoproteins at the cell surface, allowing newly formed virions to escape from the infected cells and spread to others.

Two reversible inhibitors of influenza A virus neuraminidase-Roche and Gilead Sciences Inc.'s Tamiflu oseltamivir and Relenza zanamivir from GlaxoSmithKline plc and Biota Pharmaceuticals Inc.-are marketed in the U.S. to treat and prevent influenza A. Two other reversible neuraminidase inhibitors are marketed outside the U.S.PeramiFlu peramivir from BioCryst Pharmaceuticals Inc., Green Cross Corp. and Shionogi \& Co. Ltd., and Inavir laninamivir from Daiichi Sankyo Co. Ltd. and Biota.

Although these inhibitors bind tightly to the active site of the enzyme, mutations in nearby residues of the viral protein can reduce drug affinity and even lead to resistance.

Over time, widespread use of marketed neuraminidase inhibitors could favor the spread of resistant strains, similar to what occurs with antibiotics. Indeed, Tamifluresistant influenza strains, including variants of the highly virulent $\mathrm{H} 5 \mathrm{~N} 1$ bird flu strain, have emerged in Asia in recent years.

Stephen Withers, professor of chemistry and biochemistry at the University of British Columbia, thus set out to design neuraminidase inhibitors that target the enzyme differently from the approved flu drugs.

Using a mechanism-based approach, his team designed a class of molecules based on 2,3-difluorosialic acid (DFSA), a compound that closely resembles sialic acid, the natural target of neuraminidase. DFSA, which is highly reactive, becomes covalently trapped in the enzyme's active site and eliminates the enzyme's activity.

The compounds were effective against existing resistant strains. They also could lower the odds of new resistant strains emerging,
Withers said, because the new compounds' close mimicry of the natural substrate makes it difficult for the virus to evolve resistanceassociated mutations without compromising the enzyme's normal function.

"If you minimize differences between the structure of the inhibitor and the natural substrate, it will be harder to develop resistance," said Withers. "This is also a covalent modification, which is essentially irreversible. It should thus be slower for resistance to develop."

\section{Tight fit}

The group developed a series of DFSAs with modifications designed to fit tightly into the neuraminidase active site and tested the compounds against a panel of eight clinically relevant neuraminidase variants. In vitro, the best compound had an $\mathrm{IC}_{50}$ value of less than $50 \mathrm{nM}$ for 7 of 8 neuraminidase variants, including enzymes that were resistant to Relenza and/or Tamiflu.

On the flip side, the DFSAs tested had higher in vitro $\mathrm{IC}_{50}$ values than Relenza and Tamiflu against most nonresistant strains, suggesting the new compounds could benefit from further optimization.

In a cell culture plaque-formation assay, the best new compound and Relenza showed similar prevention of viral growth. In mice infected with a nonresistant H3N3 strain, the DFSAs showed efficacy comparable to that of Relenza. The team has not yet tested the compounds in animals with resistant strains.

The compounds from Withers' team were more effective in vitro against influenza B virus neuraminidase than Relenza. Influenza B infects only humans and seals and is considered less of a public health threat than influenza $\mathrm{A}$, which infects a wide range of domestic animals as well as humans.

Findings were reported in Science.

Withers said the next step is to use a ferret model of influenza infection to test the compounds' efficacy against resistant and nonresistant strains. His team also is collaborating with the Centre for Drug Research and Development to synthesize orally available prodrug forms of the compounds.

Michael Parr, director of commercial project development at CDRD Ventures, said prior attempts by other researchers to design new inhibitors of influenza neuraminidase based on traditional models of the active site met with failure, so the approach had "fallen out of favor."

The new compounds work better than previous covalent inhibitors because of new information about the mechanism of the enzyme.

Parr said that Withers "understood the true nature of the enzyme reaction. We have capitalized on a unique mechanism of action."

Jonathon Jafari, director of business development at CDRD Ventures, said that the initial market for drugs based on Withers' compounds could be government stockpiles to combat a large-scale outbreak of virulent, drug-resistant strains.

CDRD Ventures expects that the compounds could be used as a potential second line of defense if approved drugs eventually lose their 


\section{ANALYSIS}

effectiveness. The company is investing an undisclosed amount of money in the preclinical development of the compounds.

"There's a potential for this product to be best in class, with the potential for oral availability," said Jafari.

Jafari and Parr said preclinical proof of concept should be completed this year, at which time CDRD Ventures will seek to partner or outlicense the compounds. They said another option is to raise additional venture capital or nondilutive grant funding to bring the compounds into clinical testing.

Covalent inhibitors based on Withers' DFSAs might be more potent than marketed drugs, but delivering the compounds to patients early during infection remains a challenge. In the U.S., most patients wait until the infection has nearly run its course before seeking treatment. Jafari noted that in Japan, for example, patients typically consult doctors earlier during the illness and could potentially benefit from early intervention.

Withers thinks that his compounds could be used to stop the spread of flu, not just to treat already infected patients.

"I would anticipate that this would be a prophylactic taken ahead of time if your family gets the flu or there's a pandemic," he said.

Other flu compounds in the clinic include VX-787 from Vertex Pharmaceuticals Inc. In March, Vertex reported positive results for the compound in a Phase IIa trial to treat H3N2 influenza A virus. Vertex did not disclose the target of VX-787.
The findings have been patented by the University of British Columbia, which has granted CDRD Ventures an option to license the technology.

Osherovich, L. SciBX 6(11); doi:10.1038/scibx.2013.254

Published online March 21, 2013

\section{REFERENCES}

1. Kim, J.-H. et al. Science; published online Feb. 21, 2013; doi:10.1126/science.1232552

Contact: Stephen G. Withers, The University of British Columbia, Vancouver, British Columbia, Canada

e-mail: withers@chem.ubc.ca

\section{COMPANIES AND INSTITUTIONS MENTIONED}

BioCryst Pharmaceuticals Inc. (NASDAQ:BCRX), Durham, N.C. Biota Pharmaceuticals Inc. (NASDAQ:BOTA), Rockville, Md. The Centre for Drug Research and Development, Vancouver, British Columbia, Canada

CDRD Ventures Inc., Vancouver, British Columbia, Canada Daiichi Sankyo Co. Ltd. (Tokyo:4568; Osaka:4568), Tokyo, Japan Gilead Sciences Inc. (NASDAQ:GILD), Foster City, Calif. GlaxoSmithKline plc (LSE:GSK; NYSE:GSK), London, U.K. Green Cross Corp., Yongin-Si, South Korea Roche (SIX:ROG; OTCQX:RHHBY), Basel, Switzerland Shionogi \& Co. Ltd. (Tokyo:4507; Osaka:4507), Osaka, Japan The University of British Columbia, Vancouver, British Columbia, Canada

Vertex Pharmaceuticals Inc. (NASDAQ:VRTX), Cambridge, Mass. 